\title{
STABILITY ANALYSIS FOR GENERALIZED PREDICTIVE CONTROL (GPC) WITH UNCERTAIN SYSTEMS
}

\author{
Hesham W. Gomma, MIEEE \\ Electronics, Communication and Computer Engineering Department, \\ Helwan University, Egypt
}

(Received January 15, 2006 Accepted February 27, 2006)

\begin{abstract}
This paper presents stability analysis for the well known generalized predictive control (GPC) when dealing with uncertain systems. This analysis is based on the step response coefficients and its explicit relation with the system transfer function. It reveals a part of the mystery behind the stability strength of the GPC.
\end{abstract}

KEYWORDS: Stability, Uncertain systems, Predictive control, unmodelled poles.

\section{INTRODUCTION}

The GPC is widely used in the industry despite the need of global assurance of its significant performance and stability (which is always debatable within the research society). Therefore, it cannot be claimed that the stability has reached a mature stage yet. The investigation of the GPC stability, can be traced back to Clarke et al (1987) [1], where the stability problem was approximated, under certain conditions, to the state space LQ controller. Later, using results in state space theory [2] and [3], some stability results were presented in [4] and [5]. However, the most significant contribution can be ascribed to the work presented in [6], in which, a clear representation for the closed loop system in terms of the impulse response coefficients was introduced. Reference [7] used this representation and introduced some results under certain conditions for the stability of GPC. On the other hand, reference [8] used the same principle with a different approach and referred to it: as an explicit closed loop description. A different approach [9] was introduced where a terminal state constraint is employed in linear unconstrained systems. Later, many stability results which dealt with wide range of GPC parameters were introduced in [10]. Using the explicit relation between the system transfer function and the step response coefficients, a stability analysis for the system transfer function was investigated in [11].

However, most of the aforementioned researches deal only with nominal systems and rarely with unstructured uncertainties as in [10] and [11] without paying any attention to other systems uncertainties (errors) such as unmodelled poles. Accordingly, it is useful to investigate the stability of the GPC when applied to the possible value of these errors. In this paper, the condition of GPC stability is investigated by obtaining the necessary and sufficient conditions for the roots of the characteristic equation to lie inside the unit circle. The new stability results reveal a new part of the mysteries GPC. 


\section{MODELLING OF THE SYSTEM}

The GPC approaches are applicable to both single-input/single-output (SISO) and multi-input/multi-output (MIMO) systems. In general, non-linear models can frequently be linearised around a particular operating point and described by

$$
A\left(z^{-1}\right) y(t)=B\left(z^{-1}\right) u(t-1)+C\left(z^{-1}\right) \zeta(t)
$$

where $y(t)$ is the output, $u(t)$ is the control sequence, $\zeta(t)$ is the zero mean white noise. $A, B$ and $C$ are polynomials in the backward shift operator $z^{-1}$

$$
\begin{aligned}
& A\left(z^{-1}\right)=1+a_{1} z^{-1}+a_{2} z^{-2}+\cdots \cdots+a_{n a} z^{-n a} \\
& B\left(z^{-1}\right)=b_{0}+b_{1} z^{-1}+b_{2} z^{-2}+\cdots \cdots+b_{n b} z^{-n b} \\
& C\left(z^{-1}\right)=1+c_{1} z^{-1}+c_{2} z^{-2}+\cdots \cdots+c_{n c} z^{-n c}
\end{aligned}
$$

This model is known as a CARMA (Controlled Auto-Regressive and MovingAverage) model. In industrial applications where the disturbances are non-stationary, an integral action is more appropriate [1] This will lead to automatic steady state reference setpoint tracking despite the presence of unmodelled disturbances

$$
A\left(z^{-1}\right) y(t)=B\left(z^{-1}\right) u(t-1)+C\left(z^{-1}\right) \frac{\zeta(t)}{\Delta}
$$

where $\Delta$ is the differencing operator $1-z^{-1}$. This model is known as CARIMA model (Controlled Auto-Regressive Integrated Moving-Average). For simplicity the $C$ polynomial is chosen to be 1 or $C^{-1}$ is truncated and absorbed into the $A$ and $B$ polynomials.

\section{THE OPTIMAL PREDICTION}

The main idea of GPC [1] is to find a control sequence to minimise the multistage cost function of the form:

$$
J\left(N_{1}, N_{2}, N_{u}\right)=E\left\{\sum_{j=N_{1}}^{N_{2}}[\hat{y}(t+j \mid t)-w(t+j)]^{2}+\sum_{j=1}^{N_{u}} \lambda(j)[\Delta u(t+j-1)]^{2}\right\}
$$

where $\hat{y}(t+j \mid t)$ is the predicted output $j$ steps into the future based upon information available to time $t, w(t+j)$ is the future reference trajectory, $E\{$.$\} denotes the$ expectation operator which has been used to indicate that the control values chosen are calculated conditional to the data available up to and including time $t$ and presuming the stochastic disturbance model. From the above, the tuning parameters are $N_{1}$, the minimum costing horizon, $N_{2}$, the maximum costing horizon, $\lambda(j)$, the weighting function to penalise the control sequence. In addition without loss of generality, suppose that the system has no time delay and let $N_{1}=1$ and $N_{2}=N\left(=N_{u}\right)$. 
To minimise any of the above functions the future values of the output $\hat{y}(t+j)$ should be obtained by performing long division of 1 by $\Delta A\left(z^{-1}\right)$. In fact, for long control horizon, an alternative method such as the recursion of the following Diophantine equation can be used:

$$
1=E_{j}\left(z^{-1}\right) \tilde{A}\left(z^{-1}\right)+z^{-j} F_{j}\left(z^{-1}\right)
$$

where $\tilde{A}\left(z^{-1}\right)=A\left(z^{-1}\right) \Delta$. For a unique solution the degree of the polynomials $E j$ and $F j$ should be equal to $j-1$ and $n a$ respectively. From Equation (4), it is clear by dividing 1 by $\tilde{A}\left(z^{-1}\right)$ the polynomial $E j$ is the quotient and the remainder is the factorisation of $z^{-j} F_{j}$. By multiplying each side in Equation (4) by $\Delta E_{j}\left(z^{-1}\right) z^{j}$, it is easy to see that the prediction output could be written as (see: [1])

$$
\hat{y}(t+j \mid t)=F_{j}\left(z^{-1}\right) y(t)+G_{j}\left(z^{-1}\right) \Delta u(t+j-1)
$$

where $G_{j}\left(z^{-1}\right)=E_{j}\left(z^{-1}\right) B\left(z^{-1}\right)$. From Equation (5), the optimal output predictions could be stated as:

$$
\begin{aligned}
& \hat{y}(t+1 \mid t)=G_{1} \Delta u(t)+F_{1} y(t) \\
& \hat{y}(t+2 \mid t)=G_{2} \Delta u(t+1)+F_{2} y(t) \\
& \quad \vdots \\
& \hat{y}(t+N \mid t)=G_{N} \Delta u(t+N)+F_{N} y(t)
\end{aligned}
$$

which can be written as:

$$
\begin{aligned}
& \mathbf{y}=\mathbf{G u}+\mathbf{F}\left(z^{-1}\right) y(t)+\mathbf{G}^{\prime}\left(z^{-1}\right) \Delta u(t-1) \\
& \mathbf{y}=\mathbf{G u}+\mathbf{f}
\end{aligned}
$$

where $\mathbf{f}$ is the free response and $\mathbf{G u}$ is the forced response where

$$
\begin{aligned}
& \mathbf{y}=\left[\begin{array}{llll}
\hat{y}(t+1 \mid t) & \hat{y}(t+2 \mid t) & \cdots & \hat{y}(t+N \mid t)
\end{array}\right]^{T} \\
& \mathbf{u}=\left[\begin{array}{llll}
\Delta u(t) & \Delta u(t+1) & \cdots & \Delta u(t+N-1)
\end{array}\right]^{T} \\
& \mathbf{G}^{\prime}\left(z^{-1}\right)=\left[\begin{array}{c}
\left(G_{1}\left(z^{-1}\right)-g_{0}\right) z \\
\left(G_{2}\left(z^{-1}\right)-g_{0}-g_{1} z^{-1}\right) z^{2} \\
\cdot \\
\left(G_{N}\left(z^{-1}\right)-g_{0}-g_{1} z^{-1}-\cdots-g_{N-1} z^{-(N-1)}\right) z^{N}
\end{array}\right], \mathbf{G}=\left[\begin{array}{cccc}
g_{0} & 0 & \cdots & 0 \\
g_{1} & g_{0} & \cdots & 0 \\
\vdots & \vdots & \vdots & \vdots \\
g_{N-1} & g_{N-2} & \cdots & g_{0}
\end{array}\right], \\
& \mathbf{F}\left(z^{-1}\right)=\left[\begin{array}{c}
F_{1}\left(z^{-1}\right) \\
F_{2}\left(z^{-1}\right) \\
\vdots \\
F_{N}\left(z^{-1}\right)
\end{array}\right]
\end{aligned}
$$


Now the cost function in Equation (3) and (4) can be written as:

$$
\mathbf{J}_{\text {GPC }}=(\mathbf{G u}+\mathbf{f}-\mathbf{w})^{\mathrm{T}}(\mathbf{G u}+\mathbf{f}-\mathbf{w})+\lambda \mathbf{u}^{\mathrm{T}} \mathbf{u}
$$

Minimizing the $\boldsymbol{J}$ in the above Equation (10) assuming that there are no constraints in the future, can lead to

$$
\mathbf{u}=-\left(\mathbf{G}^{\mathrm{T}} \mathbf{G}+\lambda\right)^{-1} \mathbf{G}^{\mathrm{T}}(\mathbf{f}-\mathbf{w})
$$

The first element, $\Delta u(t)$, of the matrix $\mathbf{u}$, will be applied to the system and will be repeated at every sampling period. In non-adaptive design with a time invariant model, this leads to a time invariant controller. In general, to reduce the computation needed in GPC, it is assumed that the control signals will be constant after the control horizon. The stability results of the above control law is summarised in the following sections.

\section{SYSTEMS UNCERTAINTY}

In spite of the model used in the design, there is no controller that can be considered to be robustly stable without taking into account the modelling errors (uncertainties). Hence, it is useful to design a controller which can deal with the largest possible value of these errors and which provides acceptable performance and stability. This section presents a class of theses errors called structure uncertainties which will be considered in this paper.

\section{Unmodelled Poles}

Considering that the real process has the following transfer function with extra stable poles

$$
y(t)=\frac{\widetilde{K} B\left(z^{-1}\right)}{\widetilde{A}\left(z^{-1}\right)} u(t)
$$

where $\widetilde{A}\left(z^{-1}\right)$ is the denominator of the real process that have other stable poles apart form the ones appearing in the model and $\tilde{K}$ is added so there are no discrepancies between the static gain of the process and that of the model.

\section{STEP RESPONSE COEFFICIENTS AND THE TRANSFER FUNCTION}

It should be mentioned that the step response is one of the simplest mechanisms which can be used to predict the output of a process. In this paper this mechanism will be used intensively through the introduced results. The predicted output is related to the input by the equation

$$
\hat{y}(t+k \mid t)=\sum_{i=1}^{\infty} g_{j} \Delta u(t+k-i)
$$

where $g_{i}$ are the sampled output values for the step input and $\Delta$ is the differencing operator $\left(1-z^{-1}\right)$ with $z^{-1}$ the backward shift operator. 
Lemma 1: If the system transfer function is a stable first order system that can be written as $\frac{B\left(z^{-1}\right)}{A\left(z^{-1}\right)}=\frac{b}{1-a z^{-1}}$ and $a<1$, then the system response coefficients satisfy $0 \leq g_{1} \leq g_{2} \leq \cdots g_{k} \leq \cdots \leq g_{\infty}$.

\section{Proof}

$$
\begin{aligned}
& h_{1}=b \\
& h_{2}=b a \\
& h_{3}=b a^{2} \\
& \vdots \quad \vdots \\
& h_{n}=b a^{n-1}
\end{aligned}
$$

where $h_{n}$ is the impulse response coefficient at n-instant and the step response coefficients can be written as follows

$$
\begin{aligned}
& g_{1}=h_{1} \\
& g_{2}=h_{1}+h_{2} \\
& g_{3}=h_{1}+h_{2}+h_{3} \\
& \vdots \quad \vdots \\
& g_{n}=h_{1}+h_{2}+\cdots+h_{n}
\end{aligned}
$$

It is clear that $0 \leq g_{1} \leq g_{2} \leq \cdots g_{k} \leq \cdots \leq g_{\infty}$ which completes the proof.

Lemma 2 : If the system transfer function is a stable n-order system that can be written as $\frac{B\left(z^{-1}\right)}{A\left(z^{-1}\right)}=\frac{b_{0}}{1-a_{1} z^{-1}}+\frac{b_{1}}{1-a_{2} z^{-1}}+\cdots+\frac{b_{n}}{1-a_{n} z^{-1}}$, where $b_{n}>0, n=1,2, \cdots$, then the system response coefficients satisfy $0 \leq g_{1} \leq g_{2} \leq \cdots g_{k} \leq \cdots \leq g_{\infty}$.

\section{Proof}

Using Lemma 1, it is clear that $0 \leq g_{1} \leq g_{2} \leq \cdots g_{k} \leq \cdots \leq g_{\infty}$. which completes the proof.

It should be mentioned that the above Lemmas will be the key element of the following theorems.

\section{STABILITY WITH FINITE MAXIMUM PREDICTION HORIZON}

The first theorem presents a description of the closed loop system in terms of the step response coefficients, which will be the key element in this paper. However, the main objective of the theorem is to study a popular case in GPC where the control horizon ( $N_{u}$ ) is set to one. Under this condition, the control law will be unique and can be evaluated with no matrix inversion. 


\section{Theorem 1}

If the open loop model $y(t)=\frac{B\left(z^{-1}\right)}{A\left(z^{-1}\right)} u(t)$ is stable and $y(t)=\frac{\tilde{K} B\left(z^{-1}\right)}{\widetilde{A}\left(z^{-1}\right)} u(t)$ is the real process transfer which has extra stable poles (see: Equation 12) and $N_{u}=1, N_{1}=1, N_{2}=N, \lambda=0$ and the step response coefficients of the model satisfy

$$
\begin{aligned}
& 0 \leq g_{1} \leq g_{2} \leq \cdots g_{k} \leq \cdots \leq g_{\infty} \\
& \text { and } \sum_{i=1}^{N} g_{i}^{2}>\sum_{i=1}^{N} g_{i}\left(g_{\infty}-g_{i}\right)
\end{aligned}
$$

then the closed loop system is stable.

\section{Proof}

From the assumption above it can be seen that the control horizon $N_{u}$ has been set to one. Thus, the control law can be written as

$$
\Delta u(t)=-\left(\mathbf{G}^{\mathbf{T}} \mathbf{G}\right)^{-1} \mathbf{G}^{\mathbf{T}}(\mathbf{f}-\mathbf{w})
$$

where

$$
\begin{aligned}
& \mathbf{G}=\left[\begin{array}{llll}
g_{1} & g_{2} & \cdots & g_{N}
\end{array}\right]^{T} \\
& \mathbf{f}=\left[\begin{array}{ll}
f(t+1) & f(t+2) \cdots f(t+N)
\end{array}\right] \\
& \mathbf{w}=\left[\begin{array}{ll}
w(t+1) & w(t+2) \cdots w(t+N)
\end{array}\right] \\
& \text { that is } \Delta u(t)=-\frac{\sum_{i=1}^{N} g_{i}(f(t+i)-w(t+i))}{\sum_{i=1}^{N} g_{i}^{2}}
\end{aligned}
$$

From the original algorithm the prediction output can be written as

$$
\begin{aligned}
\hat{y}(t+1) & =\sum_{i=1}^{1} g_{i} \Delta u(t+1-i)+f(t+1) \\
\hat{y}(t+2) & =\sum_{i=1}^{2} g_{i} \Delta u(t+2-i)+f(t+2) \\
\vdots & \\
\hat{y}(t+N) & =\sum_{i=1}^{N} g_{i} \Delta u(t+N-i)+f(t+N)
\end{aligned}
$$

while, by recalling the definition of the open loop step response 


$$
\hat{y}(t+k)=\sum_{i=1}^{\infty} g_{i} \Delta u(t+k-i)
$$

Therefore, if $k$ takes different values from 1 to $N$ the future outputs can be written as

$$
\begin{aligned}
& \hat{y}(t+1)=\overbrace{\sum_{i=1}^{1} g_{i} \Delta u(t+1-i)}^{\text {Forced response }}+\overbrace{\sum_{i=2}^{\infty} g_{i} \Delta u(t+1-i)}^{\text {Free response }} \\
& \hat{y}(t+2)=\sum_{i=1}^{2} g_{i} \Delta u(t+2-i)+\sum_{i=3}^{\infty} g_{i} \Delta u(t+2-i) \\
& \vdots \\
& \hat{y}(t+N)=\sum_{i=1}^{N} g_{i} \Delta u(t+N-i)+\sum_{i=N+1}^{\infty} g_{i} \Delta u(t+N-i)
\end{aligned}
$$

By comparing (20) and (22) the free response term $f$ will be represented in the following form

$$
\begin{aligned}
& f(t+1)=\sum_{i=2}^{\infty} g_{i} \Delta u(t+1-i) \\
& f(t+2)=\sum_{i=3}^{\infty} g_{i} \Delta u(t+2-i) \\
& \vdots \\
& f(t+N)=\sum_{i=N+1}^{\infty} g_{i} \Delta u(t+N-i)
\end{aligned}
$$

and from Equation (19)

$$
\sum_{i=1}^{N} g_{i}(f(t+i))=g_{1} \sum_{i=2}^{\infty} g_{i} \Delta u(t+1-i)+g_{2} \sum_{i=3}^{\infty} g_{i} \Delta u(t+2-i)+\cdots g_{N} \sum_{i=N+1}^{\infty} g_{i} \Delta u(t+N-i)
$$

Substituting Equation (24) into Equation (19) (the control law) leads to

$$
\Delta u(t)=\frac{\sum_{i=1}^{N} g_{i} \omega(t+i)}{\sum_{i=1}^{N} g_{i}^{2}+g_{1} \sum_{i=2}^{\infty} g_{i} z^{1-i}+g_{2} \sum_{i=3}^{\infty} g_{i} z^{2-i}+\cdots g_{N} \sum_{i=N+1}^{\infty} g_{i} z^{N-i}}
$$

For simplicity, let the reference be constant over the prediction horizon $\mathrm{N}$-i.e.

$$
\omega(t+1)=\omega(t+2)=\cdots \omega(t+N)=\omega
$$

Thus

$$
\sum_{i=1}^{N} g_{i} \omega(t+i)=\sum_{i=1}^{N} g_{i} \omega
$$


considering that the real process has the following transfer function with an extra

$$
y(t)=\frac{\tilde{K} B\left(z^{-1}\right)}{\tilde{A}\left(z^{-1}\right)} u(t)(\text { See Equation: } 12)
$$

Substituting Equation (24) into (27) gives

$$
y(t)=\frac{\tilde{K} B \sum_{i=1}^{N} g_{i} \omega}{\tilde{A} \Delta\left\{\sum_{i=1}^{N} g_{i}^{2}+g_{i} \sum_{i=2}^{\infty} g_{i} z^{1-i}+g_{2} \sum_{i=3}^{\infty} g_{i} z^{2-i} \cdots g_{N} \sum_{i=N+1}^{\infty} g_{i} z^{N-i}\right\}}
$$

Thus, the characteristic polynomial can be written in the following form

$$
\frac{H}{\tilde{A}}=\sum_{i=1}^{\infty} g_{i}^{2}+\sum_{i=1}^{\infty} g_{i}\left(g_{i+1}-g_{i}\right) z^{-1}+\sum_{i=1}^{\infty} g_{i}\left(g_{i+2}-g_{i+1}\right) z^{-2}+\cdots
$$

which can be written as

$$
\frac{H}{\tilde{A}}=\delta_{0}+\sum_{i=1}^{\infty} \delta_{i} z^{-i}
$$

From Rouche's theorem [12], as $\tilde{A}$ is stable, for all roots to lie in the unit circle it is sufficient that

$$
\begin{aligned}
& \left|\delta_{0}\right|>\sum_{i=1}^{\infty}\left|\delta_{i}\right| \\
& \quad \text { or }\left|\sum_{i=1}^{N} g_{i}^{2}\right|>\left|\sum_{i=1}^{N} g_{i}\left(g_{i+1}-g_{i}\right)\right|+\left|\sum_{i=1}^{N} g_{i}\left(g_{i+2}-g_{i+1}\right)\right|+\cdots
\end{aligned}
$$

As all terms in the summation are positive, thus Equation (31) holds if

$$
\sum_{i=1}^{N} g_{i}^{2}>\sum_{i=1}^{N} g_{i}\left(g_{\infty}-g_{i}\right)
$$

which is true by assumption. This completes the proof of the theorem.

It should be noticed that for a special case when $N=1$, Equation (32) can be written as the single inequality

$$
g_{1}>\frac{g_{\infty}}{2}
$$

Remark: To apply the above theorem to a non-minimum phase system where the step response will have a different behaviour, the different conditions which have been applied in [7] namely $0 \leq g_{k} \leq g_{k+1} \leq \cdots \leq g_{\infty}$ and the minimum prediction horizon $N_{1} \geq k$, should be considered. The advantage of theorem 1 is that it can be applied to many systems such as gas turbine engine, which has stable open loop and monotonic 
step response. Moreover, this theorem can provide a good explanation of the effect of the prediction horizon on the closed loop stability. More precisely, it can be seen that for large values of $N$, the summation of the left-hand side, $\sum_{i=1}^{N} g_{i}^{2}$, is bigger than the summation of the right-hand side, $\sum_{i=1}^{N} g_{i}\left(g_{\infty}-g_{i}\right)$. This occurs because the difference between $g_{i}$ and $g_{\infty}$ decreases while $g_{i}$ increases. Thus, Equation (32) holds, which guarantees the stability for all large $N$. More precisely,

$$
\begin{array}{r}
\operatorname{Lim}_{N \rightarrow \infty} \frac{1}{N} \sum_{i=1}^{N} g_{i}^{2}=g_{\infty}^{2}>\operatorname{Lim}_{N \rightarrow \infty} \frac{1}{N} g_{\infty} \sum_{i=1}^{N}\left(g_{\infty}-g_{i}\right) \\
>\operatorname{Lim}_{N \rightarrow \infty} \frac{1}{N} \sum_{i=1}^{N} g_{i}\left(g_{\infty}-g_{i}\right)
\end{array}
$$

\section{Theorem 2}

If the open loop model $y(t)=\frac{B\left(z^{-1}\right)}{A\left(z^{-1}\right)} u(t)$ is stable and $y(t)=\frac{\tilde{K} B\left(z^{-1}\right)}{\widetilde{A}\left(z^{-1}\right)} u(t)$ is the real process transfer which has extra stable poles (see: Equation 12), and $N_{u}=1, N_{1}=1, N_{2}=N$ and $\lambda(i)=\lambda$. Assume also that the step response coefficients satisfy $0 \leq g_{1} \leq g_{2} \leq \cdots g_{k} \leq \cdots \leq g_{\infty}$,

$$
\sum_{i=1}^{N} g_{i}\left(g_{i+1}-g_{i}\right) \geq \lambda
$$

and

$$
\sum_{i=1}^{N} g_{i}^{2}+2 \lambda>\sum_{i=1}^{N} g_{i}\left(g_{\infty}-g_{i}\right)
$$

Then the closed loop system is stable.

\section{Proof}

By using Equations (11) and (13), the control law can be written as

$$
\begin{gathered}
\Delta u(t)=-\frac{\sum_{i=1}^{N} g_{i}(f(t+i)-w(t+i))}{\sum_{i=1}^{N} g_{i}^{2}+\lambda} \\
\Delta u(t)=-\frac{\sum_{i=1}^{N} g_{i}(f(t+i)-w(t+i))}{\left(\sum_{i=1}^{N} g_{i}^{2}+\lambda\right)}
\end{gathered}
$$


From the control law it is clear that

$$
\Delta u(t)=\frac{\sum_{i=1}^{N} g_{i} \omega(t+i)}{\left(\sum_{i=1}^{N} g_{i}^{2}+\lambda\right)+g_{1} \sum_{i=2}^{\infty} g_{i} z^{1-i}+g_{2} \sum_{i=3}^{\infty} g_{i} z^{2-i}+\cdots g_{N} \sum_{i=N+1}^{\infty} g_{i} z^{N-i}}
$$

Following the same procedures of the above theorem and applying Rouche's theorem, for closed loop poles to lie in the unit circle it is sufficient that

$$
\left|\sum_{i=1}^{N} g_{i}^{2}+\lambda\right|>\left|\sum_{i=1}^{N} g_{i}\left(g_{i+1}-g_{i}\right)-\lambda\right|+\left|\sum_{i=1}^{N} g_{i}\left(g_{i+2}-g_{i+1}\right)\right|+\cdots \cdots
$$

By the assumption (Equation 13), all elements are positive and hence the inequality holds if

$$
\left|\sum_{i=1}^{N} g_{i}^{2}+\lambda\right|>\left|\sum_{i=1}^{N} g_{i}\left(g_{\infty}-g_{i}\right)-\lambda\right|
$$

or

$$
\sum_{i=1}^{N} g_{i}^{2}+2 \lambda>\sum_{i=1}^{N} g_{i}\left(g_{\infty}-g_{i}\right)
$$

which completes the proof.

Remark: From Equation (41) it can be seen that $\lambda$ is a very influential parameter in the system stability, where increasing $\lambda$ by a reasonable amount to satisfy the conditions stated above (Equations 35 and 41) can lead to the stability of the closed loop system. Moreover, this theorem is essential in giving an interpretation for the effect of $\lambda$ on the system performance/robustness. From Equation (15), it is clear that the gain of the control law increases as $\lambda$ decreases, which in turn improves the performance and diminishes the robustness. However, it is still worth mentioning that, from Equation (35), the maximum value of $\lambda$ which can be used in the theorem is bounded by

$$
\begin{aligned}
\lambda_{\max }=\sum_{i=1}^{N} g_{i}\left(g_{i+1}-g_{i}\right)< & \sum_{i=1}^{N} g_{\infty}\left(g_{i+1}-g_{i}\right) \\
& <g_{\infty}\left(g_{N+1}-g_{1}\right) \\
& <g_{\infty}\left(g_{\infty}-g_{1}\right)
\end{aligned}
$$

It is clear that increasing the maximum prediction horizon can allow larger values of $\lambda$ to be used with guaranteed stability. However, the effect of an infinite maximum prediction horizon will be investigated in more detail, in the following section. 


\section{LARGE VALUES OF MINIMUM AND MAXIMUM PREDICTION HORIZON}

In this section the idea of designing a controller with a very large minimum prediction horizon will be considered. Similar conditions were presented by [7]; however, it cannot be considered as a precise result due to the approximations which were used in developing the proof. In the mean time, uncertainties were not considered Thus, the next theorem will shed some light on the effect of setting the minimum prediction horizon to a relatively large value with application to uncertain systems.

\section{Theorem 2}

If the open loop model $y(t)=\frac{B\left(z^{-1}\right)}{A\left(z^{-1}\right)} u(t)$ is stable and $y(t)=\frac{\tilde{K} B\left(z^{-1}\right)}{\widetilde{A}\left(z^{-1}\right)} u(t)$ is the real process transfer which has extra stable poles (see: Equation 12), $N_{2}>N_{1}, N_{u}=1$, $\lambda=0$ and the step response coefficients achieve

$$
0 \leq g_{N_{1}} \leq \cdots \leq g_{N_{2}} \leq \cdots \leq g_{\infty}
$$

then the closed loop system is stable for all large enough values of $N_{1}$.

\section{Proof}

From Equation (18), the control law can be represented as

$$
\Delta u(t)=-\frac{\sum_{i=N_{1}}^{N_{2}} g_{i}(f(t+i)-w(t+i))}{M}
$$

where $M=\sum_{i=N_{1}}^{N_{2}} g_{i}^{2}$

Similar to Equation (37), Equation (44) can be written in the following form

$$
\begin{gathered}
\Delta u(t)\left\{1+\frac{1}{M}\left[g_{N_{1}} \sum_{i=N_{1}+1}^{\infty} g_{i} z^{N_{1}-i}+g_{N_{1}+1} \sum_{i=N_{1}+2}^{\infty} g_{i} z^{N_{1}+1-i}\right.\right. \\
\left.\left.+\cdots g_{N_{2}} \sum_{i=N_{2}+1}^{\infty} g_{i} z^{N_{2}-i}\right]\right\}=\frac{1}{M} \sum_{i=N_{1}}^{N_{2}} g_{i} \omega
\end{gathered}
$$

Substituting Equation (46) into Equation (44) gives the characteristic polynomial

$$
\frac{H}{\tilde{A}}=1+\left[\left(\frac{1}{M} \sum_{i=1}^{\infty} g_{i} g_{i+1}\right)-1\right] z^{-1}+\left[\frac{1}{M} \sum_{i=1}^{\infty} g_{i}\left(g_{i+2}-g_{i+1}\right)\right] z^{-2}+\cdots
$$

For stability (Rouche's Theorem), it is sufficient that

$$
1>\left|\left(\frac{1}{M} \sum_{i=N_{1}}^{N_{2}} g_{i} g_{i+1}\right)-1\right|+\left|\frac{1}{M} \sum_{i=N_{1}}^{N_{2}} g_{i}\left(g_{i+2}-g_{i+1}\right)\right|+\cdots
$$




$$
\text { As }\left(\frac{1}{M} \sum_{i=N_{1}}^{N_{2}} g_{i} g_{i+1}\right)-1 \geq 0 \text { and } g_{i}\left(g_{i+k+1}-g_{i+k}\right)>0
$$

where $k=1,2, \cdots$, the condition reduces to

$$
\left|\left(\frac{1}{M} \sum_{i=N_{1}}^{N_{2}} g_{i} g_{\infty}\right)-1\right|<1
$$

which is valid for all large enough $N_{1}$ as $\lim _{N_{1} \rightarrow \infty} \frac{\sum_{i=N_{1}}^{N_{2}} g_{i} g_{\infty}}{M}=1$

Thus the system is stable and the proof is completed.

Remark: Theorem 2 differs from the one developed by [7] in which the control strategy of a stable open loop system was considered to tend towards a mean-level law. To reach this conclusion, reference [7] ignored the coefficients of the higher order of the shift operator $\left(z^{-1}\right)$ (see: Equation 39), they considered the differences between $g_{i+k+1}$ and $g_{i+k}$, in the summation $\sum_{i=N_{1}}^{N_{2}} g_{i}\left(g_{i+k+1}-g_{i+k}\right)$ goes to zero, when $N_{1} \rightarrow \infty$, which means ignoring any residual errors could appear. In addition, reference [7] did not consider any system uncertainties. Therefore to avoid this approximation and to prove the closed loop stability, in Theorem 2, Rouche's theorem has been used. In addition to the above results which have been based on Rouche's theorem, new results will be introduced. The following condition is based on Jury's table, when [8] has shown (based on [13]) that by using Hurwitz's Theorem in complex analysis, given $L\left(z^{-1}\right)=1+L_{1} z^{-1}+L_{2} z^{-2}+\cdots$, as a characteristic equation, if $1>L_{1}>L_{n}>\cdots>0$ then $L\left(z^{-1}\right)$ will never equal to zero when $|z| \geq 1$, i.e. $L\left(z^{-1}\right)$ is stable. It is clear that more stability results can be obtained by the using the above conditions.

\section{Theorem 3}

If the open loop model $y(t)=\frac{B\left(z^{-1}\right)}{A\left(z^{-1}\right)} u(t)$ is stable and $y(t)=\frac{\widetilde{K} B\left(z^{-1}\right)}{\widetilde{A}\left(z^{-1}\right)} u(t)$ is the real process transfer which has extra stable poles (see: Equation 12) and step response coefficients have the following relation

$$
\sum_{i=1}^{N} g_{i}^{2}>\sum_{i=1}^{N} g_{i}\left(g_{i+1}-g_{i}\right)>\sum_{i=1}^{N} g_{i}\left(g_{i+2}-g_{i+1}\right)>\cdots \cdots>0
$$

then choices $N_{u}=1, N_{1}=1, N_{2}=N$ and $\lambda=0$ results in closed loop stability. 


\section{Proof}

From [8] and by recalling Equation (29), the closed loop system is stable (all roots within the unit circle) if

$$
\sum_{i=1}^{N} g_{i}^{2}>\sum_{i=1}^{N} g_{i}\left(g_{i+1}-g_{i}\right)>\sum_{i=1}^{N} g_{i}\left(g_{i+2}-g_{i+1}\right)>\cdots>0
$$

which completes the proof.

\section{STABILITY WITH CONTROL HORIZON GREATER THAN OR EQUAL TO ONE $\left(N_{u} \geq 1\right)$}

The reader's attention is now brought to a common case in GPC, where the control horizon is chosen to have values greater than $\left(N_{u} \geq 1\right)$. Although the increase in the value of $N_{u}$ increases the amount of computation required, it is still preferable in most applications, as it provides better performance with high optimality to the GPC [1]. The following theorems present new sufficient conditions for stability with a special case of $N_{u} \geq 1$ when systems uncertainties are considered. The stability of this case has been tackled before considering only nominal models ([1], [14], and [3]), however, none of them have dealt with the problem through an explicit representation of the closed loop system. The stability was proved by observing that the predictive scheme in question tends to the steady-state LQ controller for which there is a stability guarantee or by using the monotonicity of the optimal cost function such as [15] and [11]. It should be mentioned that the main difficulty in finding a clear expression for the closed loop system is the presence of the square matrix $\left(N_{u} \times N_{u}\right)$ inversion. Thus, the following theorem will try to approach this problem through certain conditions considering system uncertainties.

\section{Theorem 4}

In GPC, if the open loop model $y(t)=\frac{B\left(z^{-1}\right)}{A\left(z^{-1}\right)} u(t)$ is stable and $y(t)=\frac{\tilde{K} B\left(z^{-1}\right)}{\widetilde{A}\left(z^{-1}\right)} u(t)$ is the real process transfer which has extra stable poles such as $\widetilde{A}\left(z^{-1}\right)=A^{+}\left(z^{-1}\right) A\left(z^{-1}\right)$ (see: Equation 12$)$ where $A^{+}\left(z^{-1}\right)$ is a polynomial that contains the unmodelled stable poles then choices $N_{1}=1,\left(N_{2}-N_{1}+1\right)=N_{u}=N$ and $\lambda=0$, results in closed loop stability.

\section{Proof}

As the weighting function $\lambda$ is selected to be equal to zero, the GPC control law can be written in the following form:

$$
\mathbf{u}=-\underbrace{\left(\mathbf{G}^{\mathrm{T}} \mathbf{G}\right)^{-1}}_{\mathbf{P}} \mathbf{G}^{\mathbf{T}}(\mathbf{f}-\mathbf{W})
$$

As $\mathbf{G}$ is a square matrix then for all $\mathbf{G}$ : 


$$
\mathbf{P}=\left(\mathbf{G}^{\mathrm{T}} \mathbf{G}\right)^{-\mathbf{1}} \mathbf{G}^{\mathrm{T}}=\mathbf{G}^{-\mathbf{1}}
$$

where the matrix $\mathbf{G}$ is a square matrix consists of the plants's step responses $\left(g_{i}\right)$ (see: Equation 9).Thus, the control law can be given in the following form

$$
g_{1}^{N} \Delta u(t)=-g_{1}^{N-1}(f(t+1)-w(t+1))
$$

Let the reference trajectory be constant over the prediction horizon, Equation 47 can be written as

$$
g_{1} \Delta u(t)=-\sum_{i=2}^{\infty} g_{i} \Delta u(t+1-i)+\omega
$$

which can be written

$$
\Delta u(t)=\frac{\omega}{g_{1}+\sum_{i=2}^{\infty} g_{i} z^{1-i}}
$$

Recalling the system transfer function

$$
y(t)=\frac{\tilde{K} B\left(z^{-1}\right)}{\widetilde{A}\left(z^{-1}\right)} u(t)
$$

Consequently, substituting Equation (57) into (58) leads to the following closed loop system

$$
y(t)=\frac{B \omega}{\tilde{A} \Delta\left(g_{1}+\sum_{i=2}^{\infty} g_{i} z^{1-i}\right)}
$$

From the above, the characteristic equation can be written as

$$
\frac{H}{\tilde{A}}=\Delta\left(g_{1}+\sum_{i=2}^{\infty} g_{i} z^{1-i}\right)=g_{1}+\left(g_{2}-g_{1}\right) z^{-1}+\left(g_{3}-g_{2}\right) z^{-2}+\cdots
$$

If the step response can be written as

$$
y(t)=\sum_{i=1}^{\infty} g_{i} \Delta u(t-i)
$$

Thus the relation between the step responses and plant transfer function can be written as

$$
\frac{B\left(z^{-1}\right)}{A\left(z^{-1}\right)}=g_{1} z^{-1}+\left(g_{2}-g_{1}\right) z^{-2}+\left(g_{3}-g_{2}\right) z^{-3} \cdots
$$

But, from Equation (61), the closed loop characteristic equation can be written as

$$
\frac{H}{\widetilde{A}}=g_{1}+\left(g_{2}-g_{1}\right) z^{-1}+\left(g_{3}-g_{2}\right) z^{-2} \cdots
$$


By comparing Equations (63) and (64), it can be found that

$$
H\left(z^{-1}\right) z^{-1}=B\left(z^{-1}\right) A^{+}\left(z^{-1}\right)
$$

By recalling the closed loop transfer function Equation (52), and substituting from Equation (65)

$$
y(t)=\frac{1}{z A^{+}\left(z^{-1}\right)} \omega(t)
$$

From the above conditions, it is clear that the closed loop systems is stable which completes the proof.

\section{Theorem 5}

In GPC, assume the open loop model $y(t)=\frac{B\left(z^{-1}\right)}{A\left(z^{-1}\right)} u(t)$ is stable with a monotonic step response i.e.

$$
0 \leq g_{1} \leq g_{2} \leq \cdots g_{k} \leq \cdots \leq g_{\infty}
$$

and $y(t)=\frac{\tilde{K} B\left(z^{-1}\right)}{\tilde{A}\left(z^{-1}\right)} u(t)$ is the real process transfer which has extra stable poles (see: Equation 12), if $N_{1}=1, N_{u}=\left(N_{2}-N_{1}+1\right)=N, \lambda=0$, and $g_{1}>\frac{g_{\infty}}{2}$, then the closed loop system is stable.

\section{Proof}

Similar to the above, from Rouche's theorem [12], as $A$ is stable, in Equation (56) for all roots to lie in the unit circle it is sufficient that

$$
g_{1}>\left(g_{\infty}-g_{1}\right)
$$

or alternatively

$$
g_{1}>\frac{g_{\infty}}{2}
$$

which completes the proof.

\section{Theorem 6}

In GPC, assume the open loop model $y(t)=\frac{B\left(z^{-1}\right)}{A\left(z^{-1}\right)} u(t)$ has a convex step response such that $g_{1}>\left(g_{2}-g_{1}\right)>\left(g_{3}-g_{2}\right) \cdots>0$ and $y(t)=\frac{\tilde{K} B\left(z^{-1}\right)}{\tilde{A}\left(z^{-1}\right)} u(t)$ is the real 
process transfer which has extra stable poles (see: Equation 12), then if $N_{1}=1$, $\left(N_{2}-N_{1}+1\right)=N, \lambda=0$, the closed loop system is stable.

\section{Proof}

Following the proof of Theorem 4, from Equation (55), the characteristic polynomial can be written as:

$$
\frac{H}{A}=g_{1}+\left(g_{2}-g_{1}\right) z^{-1}+\left(g_{3}-g_{2}\right) z^{-2} \cdots
$$

Thus, from the sufficient condition in [8], the closed loop system will be stable if:

$$
g_{1}>\left(g_{2}-g_{1}\right)>\left(g_{3}-g_{2}\right) \cdots>0
$$

Which completes the proof.

Remark: It is important to note that many systems such as gas turbines have a convex step response [10]. Accordingly, the significant of the above theorems (Theorems 5 and 6) that they have a great importance in GPC stability as they show new sufficient condition where the system can be stable when $\left(N_{2}-N_{1}+1\right)=N_{u}=N \geq 1$. Furthermore, it should be noticed that when the above theorems are applied to systems with time delay $(d)$, the minimum prediction horizon $\left(N_{1}\right)$ can be selected such as $N_{1}=d+1$.

\section{CONCLUSION}

In this paper various stability results for GPC algorithms when deals with uncertain systems namely unmodelled poles are presented. In contrast to most existing theorems, which relied on state space representation or the monotonicity decrease of the receding cost function (without paying attention to uncertain systems), this paper introduced new theorems using an explicit representation for the closed loop system. These results cover many sufficient conditions for stability, of uncertain systems (unmodelled poles) which can be considered as design guidelines for plants with monotonic or convex step responses such as gas turbine engines [10]. In general, the analyses are based on representing the closed loop system in terms of the step response coefficients. This concept can be extended to cover other forms of uncertainties which will be considered in future research. However, it should be mentioned that the complexity of developing a general stability theorem for the aforementioned algorithms could be attributed to the complexity of the square inversion matrix which involves all system's parameters.

\section{REFERENCES}

[1] Clarke, D.W., Mohtadi C., and Tuffs P.S., "Generalized predictive control-part I and II", Automatica, Vol. 23, No.2, pp.137-160, 1987.

[2] Kwon, W.H. and Pearson A.E., "On the stabilisation of a discrete constant linear system", IEEE Transactions on Automatic Control, Vol. 20, No. 6, pp. 800-801, 1975. 
[3] Kwon, W.H., and Byun, D.G., "Receding horizon tracking control as a predictive control and its stability properties", International Journal of Control, Vol.50, No.5, pp.1807-1824, 1989.

[4] Clarke, D.W. and Scattolini, R., "Constrained receding horizon predictive control”, Proc. IEE-D, Vol. 138, No.4, pp.347-354, 1991.

[5] Mosca, E. and Zhang, J., "Stable receding of predictive control", Automatica, Vol.28, No.6, pp.1229-1233, 1992.

[6] De Nicolao., G and Scattolini, R., "Advances in model predictive control, chapter stability and output terminal constraints in predictive control", Oxford University Press, 1994.

[7] Yoon T.W. and Clarke, D.W., "A reformulation of receding-horizon predictive control", International Journal System Science, Vol. 26, No. 7, pp. 1383-1400, 1995.

[8] Zhang, J., and Xi, Y., "Some Stability results', International Journal of control", Vol.70, No.5, pp.831-840, 1998.

[9] Bemporad, A., Chisci, L., and Mosca, E. "On the stabilizing property of SIORHC”. Automatica, 30, 2013-2015, 1995.

[10] Gomma H.W., "Robust and predictive control of 1.5 MW gas turbine engine". PhD Thesis, Exeter University, UK, 1999.

[11] Gomma, H. W., H. Yu and T. C. Yang "Generalized Predictive Control: Stability Note", Proceeding of the 10th Chinese Automation \& Computing Society Conference in the UK, Liverpool, England, pp.25-29, 2004.

[12] Spiegel, M. R., "Complex variables", McGraw-Hill, 1964.

[13] Jury, E. I., "Theory and application of z-transform method", Wley, 1964.

[14] Clarke D.W., and Mohtadi, C., "Properties of generalized predictive control", Automatica, Vol.25, No.6, pp.859-875, 1989

[15] Rawlings, J. B., and Muske, K. R., "The stability of constrained receding horizon control”, IEEE Trans. AC, 38(10): 1512-1516, 1993.

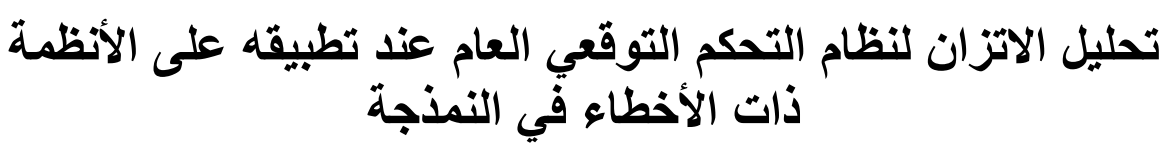

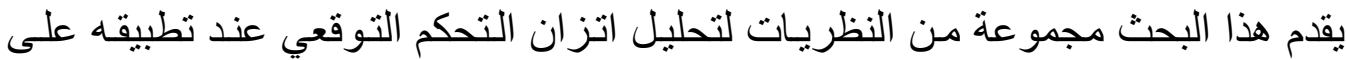

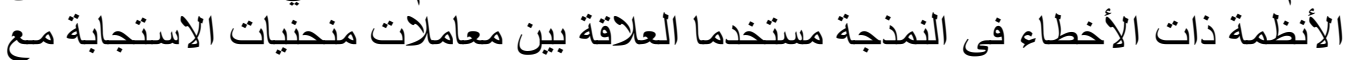

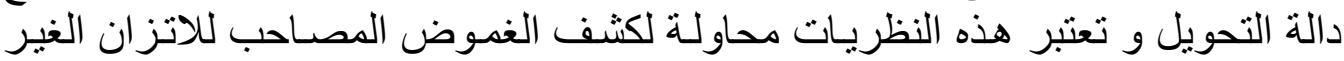
كامل التفسير عند تطبيق لنظام التحكم التوقعي العام مع العديد من النظم . لنّات 